\title{
Laparoscopic subtotal hysterectomy versus laparoscopic total hysterectomy: a decade of experience
}

\author{
J. S. van Evert • J. M. J. Smeenk • \\ F. P. H. L. J. Dijkhuizen • J. H. de Kruif • K. B. Kluivers
}

Received: 29 May 2009/Accepted: 21 September 2009/Published online: 14 November 2009

(C) The Author(s) 2009. This article is published with open access at Springerlink.com

\begin{abstract}
At present, there are only few data on the surgical outcomes of laparoscopic hysterectomy (LH). Up till now, it has been unclear whether there is a difference in number of complications among the subcategories of laparoscopic total hysterectomy and laparoscopic subtotal hysterectomy (LSH). Therefore, we have performed a retrospective analysis to evaluate the peri- and postoperative outcomes in women undergoing LSH versus LH. This multi-centre retrospective cohort study (Canadian Task Force classification II-2) was conducted in multi-centres (two teaching hospitals and one university medical centre) in the Netherlands, all experienced in minimally invasive gynaecology. In a multi-centre retrospective cohort study we compared the long-term outcomes of laparoscopic subtotal hysterectomy and laparoscopic total hysterectomy (including laparoscopic assisted vaginal hysterectomy, laparoscopic hysterectomy and total laparoscopic hysterectomy). All laparoscopic hysterectomies
\end{abstract}

Précis Laparoscopic subtotal hysterectomy as compared with the different types of laparoscopic total hysterectomy is associated with more long-term postoperative complications, whereas laparoscopic total hysterectomy is associated with more short-term complications.

All authors have made substantial contributions to the manuscript.

J. S. van Evert $(\bowtie) \cdot$ F. P. H. L. J. Dijkhuizen

Rijnstate hospital,

Wagnerlaan 55,

6815 AD Arnhem, The Netherlands

e-mail: j.vanevert@obgyn.umcn.nl

J. M. J. Smeenk · J. H. de Kruif

Canisius Wilhelmina hospital,

Nijmegen, The Netherlands

K. B. Kluivers

Radboud University Nijmegen Medical Centre,

Nijmegen, The Netherlands from the last 10 years (January 1998 till December 2007) were included. Patient characteristics, intra- and postoperative complications, operating time and duration of hospital stay were recorded. The minimum follow-up was 6 months. A total of 390 cases of laparoscopic hysterectomies were included in the analysis: 192 laparoscopic subtotal hysterectomies and 198 laparoscopic total hysterectomies. Patient characteristics such as age and parity were equal in the groups. The overall number of short-term and long-term complications was comparable in both groups: $17 \%$ and $15 \%$. Short-term complications (bleeding, fever) were $3 \%$ in the LSH group and $12 \%$ in the LH group. Long-term complications were (tubal prolapse and cervical stump reoperations) $15 \%$ in the $\mathrm{LSH}$ group and $3 \%$ in the $\mathrm{LH}$ group. Laparoscopic subtotal hysterectomy as compared with the different types of laparoscopic total hysterectomy is associated with more long-term postoperative complications, whereas laparoscopic total hysterectomy is associated with more short-term complications.

Keywords Laparoscopic hysterectomy .

Laparoscopic subtotal hysterectomy . Complications

\section{Introduction}

The technique of laparoscopic hysterectomy (LH) offers some clear advantages over abdominal hysterectomy ( $\mathrm{AH})$. LH is associated with a reduction in blood loss, fewer wound infections, less postoperative pain, a shorter hospital stay and quicker return to normal activities. However, LH is associated with longer operating time and a greater risk of damaging the bladder or ureter as compared to AH [1-3]

Total versus subtotal hysterectomy were compared in a meta-analysis by Lethaby et al. This review has not 
confirmed the perception that subtotal hysterectomy offers improved outcomes for sexual, urinary or bowel function when compared with total abdominal hysterectomy. Surgery is shorter, and intraoperative blood loss and fever are reduced, but women are more likely to experience ongoing cyclical bleeding up to a year after surgery with subtotal hysterectomy compared to total hysterectomy [4].

Some argue that the benefits of LH may be even more apparent with laparoscopic subtotal hysterectomy (LSH) as compared with one of the three categories of LH. These benefits may be associated with a more rapid improvement in short-term quality of life measures. Furthermore, it is suggested that the LSH procedure is less invasive, easier to perform and carries a lower risk of infections and urinary tract lesions and might be feasible in a day-care setting. On the other hand, LSH is associated with persistent vaginal bleeding and more hospital readmissions [5].

Up till now, it has been unclear whether one of the subcategories of LH or LSH is associated with more or less complications. At present, there are only few data on the surgical outcomes of LH. Therefore, we performed a retrospective analysis to evaluate the peri- and postoperative outcomes in women undergoing a LSH versus a LH.

\section{Materials and methods}

This multi-centre retrospective study was conducted in three hospitals in the east of the Netherlands. Two large teaching hospitals: Canisius Wilhelmina Hospital in Nijmegen and Rijnstate Hospital in Arnhem and one university hospital: Radboud University Nijmegen Medical Centre. All LH and LSH from January 1998 till December 2007 were included for both benign as well as malign indication. Information was obtained from medical records about patient characteristics (indication, age and parity), surgery (operating time, blood loss, complications and conversions) and the postoperative period.

Since the documentation of the specifics of the laparoscopic procedure was not conclusive on the matters of ligation of the uterine vessels by laparoscopy or vaginally and the differences in interpretation of the classification, we combined all laparoscopic total hysterectomies into one group (LH) and did not make a classification as described by Reich et al. [6]. The surgical technique of the LH has been described before [6]. For LSH, amputation of the cervix was performed by either a unipolar Lina loop or Lap Loop.

Complications were classified in short-term and longterm complications. Short-term complications were defined as blood loss, urinary tract infection, vaginal vault haematoma, ureter lesions, fever $>38.5$ C. Long-term complications were defined as: tubal prolapse, persistent vaginal bleeding abdominal wall problems, dyspareunia, postoperative pelvic pain and pelvic organ prolapse. Conversions (laparoscopy to laparotomy) were not regarded as complications but were described separately as a standard of prudent surgery.

All data were analysed in Statistical Package for Social Sciences, version 16.0 (SPSS, Inc., Chicago, IL). To compare normally distributed continuous data from two groups, we analysed using a two-sided independent samples; Student's $T$ tests and $Z$ tests were used for categorical data. $P$ values lower than 0.05 were accepted as statistically significant.

\section{Results}

During the study period, 396 laparoscopic hysterectomies were performed; of which, six were laparoscopic Wertheim procedures and were excluded. Thus, 390 patients were included in the study; of which, 192 were laparoscopic subtotal hysterectomies, and 198 were laparoscopic total hysterectomies. Fifty total laparoscopic hysterectomies were performed for low-grade endometrium carcinoma. The groups showed no statistically significant differences concerning parity or indication for surgery (Table 1). The women in the LH group were significantly older as compared with the LSH group, 49 years (30-81 years) versus 44 years (28-60 years) respectively. The operating time and blood loss were not significantly different in the two groups.

The total number of complications was $34(17 \%)$ in the LSH group and $30(15 \%)$ in the LH group (Table 2) Complications during surgery were seen more often in the LH group. Furthermore, more vaginal vault haematoma were seen in the LH group. The vaginal vault was closed vaginally in most cases; this may be an explanation for the infected haematoma. No difference was found in the use of antibiotics during surgery.

One tubal prolapse occurred, in a case where a total laparoscopic hysterectomie (TLH) had been performed, with laparoscopic closure of the vault.

The higher rate of anaesthesia related problems in the LH group may be related to the patient characteristics. The LH group was significantly older, which can be explained by the inclusion of malignant cases.

One ureter lesion in a LH case was identified 2 weeks after the operation because of vaginal urine loss. The patient was reoperated for ureter reimplantation per laparotomy. The recovery was uneventful.

The number of conversions is higher in the LSH group, and most conversions were performed because of technical problems, three of them were due to haemorrhage, one was due to a defect morcellator.

In the LSH group, cervical stump problems immediately draw attention. Six percent (12 patients) of the patients complained of persistent vaginal bleeding. Four patients 
Table 1 Indications for hysterectomy by treatment group

Data presented as absolute numbers and percentage LSH laparoscopic subtotal hysterectomy, $L H$ laparoscopic (total) hysterectomy

\begin{tabular}{lll}
\hline Indication & LSH $(n=192)$ & LH $(n=198)$ \\
\hline Menorrhagia & $92(48 \%)$ & $71(36 \%)$ \\
Metrorrhagia & $47(25 \%)$ & $27(14 \%)$ \\
Dysmenorrhoea & $20(10 \%)$ & $17(9 \%)$ \\
Uterus myomatosus mechanical complaints & $32(17 \%)$ & $18(9 \%)$ \\
Malignancy & - & $50(25 \%)$ \\
Other & $1(0.5 \%)$ & $15(8 \%)$ \\
\hline
\end{tabular}

needed further surgery to remove the cervix. In the other cases, coagulation of the cervix, or an expectant policy, was performed. Dyspareunia was described more often in the LSH group.

\section{Discussion}

We conducted a retrospective cohort study to evaluate the outcomes of different types of laparoscopic total hysterectomy versus LSH.

Table 2 Short-term and long-term complications by treatment group

\begin{tabular}{|c|c|c|c|}
\hline Complications & LSH (192) & LH (198) & $Z$ value \\
\hline Short term & $7(3 \%)$ & $24(12 \%)$ & 3.15 \\
\hline Blood loss $<1 \quad \mathrm{~L}$ & $3(2)$ & $6(3)$ & NS \\
\hline Blood loss $>1 \quad L$ & $1(0.5)$ & $3(1.5)$ & NS \\
\hline Urinary tract infection & $2(1)$ & $1(0.5)$ & NS \\
\hline Fever & $1(0.5)$ & $2(1)$ & NS \\
\hline Anaesthesia-related $^{\mathrm{b}}$ & - & $3(1.5)$ & NS \\
\hline Vault haematoma & - & $9(4.5)$ & 2.66 \\
\hline Long term & $27(15)$ & $6(3)$ & 3.97 \\
\hline Cervical stump problem & $12(6)$ & - & 3.30 \\
\hline Excision of the cervix & $4(2)$ & - & 1.54 \\
\hline Dyspareunia & $4(2)$ & - & 1.54 \\
\hline Ureter lesion & - & $1(0.5)$ & NS \\
\hline Tubal prolapse & - & $1(0.5)$ & NS \\
\hline Abdominal wall prob ${ }^{\mathrm{a}}$ & $2(1)$ & $1(0.5)$ & NS \\
\hline Urinary incontinence & $2(1)$ & $2(1)$ & NS \\
\hline Pelvic pain & $3(2)$ & $1(0.5)$ & 1.52 \\
\hline Conversion & $9(5)$ & $3(1.5)$ & NS \\
\hline Technical problem & 5 & 1 & NS \\
\hline
\end{tabular}

Data presented as absolute numbers and percentage; $\mathrm{NS}=\mathrm{No}$ statistically significant difference between the groups; $p$ value $=p$ value for difference between groups using $t$ test in case of normal distributed variables and Mann-Whitney test in case of non-normal distributed variables

LSH laparoscopic subtotal hysterectomy, $L H$ laparoscopic hysterectomy

${ }^{a}$ Two abdominal wall haematoma and a hernia cicatricialis: needing surgery

${ }^{\mathrm{b}}$ One atrialfibrillation, one anti-cholinerg syndrome, one decompensatio cordis
LSH was associated with more long-term complications such as cervical stump problems and dyspareunia, whereas LH was associated with more short-term complications: urinary tract lesions, more blood loss and more postoperative infections.

A retrospective cohort study has its limitations. We are aware that we are not completely informed about the influence of several possible confounders as body mass index, smoking, and previous surgery. The relatively large sample size may compensate this limitation.

In the LSH group, we found more problems of persistent vaginal bleeding. A reoperation (removal of the cervix) was necessary in $2 \%$ of the women. Lieng evaluated in a retrospective postal questionnaire the long-term outcomes following LSH. About $24 \%$ of the women reported experiencing vaginal bleeding up to 3 years following their $\mathrm{LSH}$, although this was rated as a small amount of blood loss in $90 \%$ of cases. Only $7 \%$ of the women had further related surgery after the LSH procedure. Similar to our results, extirpation of the cervix was necessary in $2 \%$ of the patients [5].

Donnez suggested that uterine amputation above the level of the internal cervical ostium might be a cause for the high occurrence of vaginal bleeding following LSH [7]. Lieng showed a significantly higher rate of postoperative vaginal bleeding in patients treated by less experienced surgeons [5]. In our study, we can also find an association between the experience of the surgeon and cervical problems. All cases of surgery for persistent vaginal bleeding were operated by surgeons who had performed less than 50 of these procedures.

In our study group, we did not find any patients with deep endometriosis. In both groups, the patients with adenomyosis or endometriosis are included in the group of patients with dysmenorrhoea.

Dyspareunia and abdominal pain was reported by $4 \%$ of patients after LSH. All these patients were operated because of mechanical complaints of a uterus myomatosus. Although the overall group of women after LSH reported a significant decrease in pain, women having a LSH because of endometriosis reported significantly higher levels of pain after surgery [5]. It has been suggested that LSH may not be the first choice procedure in the presence of preoperative 
pelvic pain and/or endometriosis due to the risk of persistent pelvic pain [8].

El-Touky found no negative impact on sexual function 6 months after LSH or TLH [9]. In our results, more patients experienced dyspareunia in the LSH group.

The amount of conversions is higher in de LSH group, but selection bias may play a role. In cases with presumed complicated hysterectomies, LSH may have been preferred over LH.

In the LH group, one ureter lesion has occurred, which was discovered 2 weeks after surgery because of vaginal urine loss. Meta-analysis of randomised studies of laparoscopic versus abdominal hysterectomy has shown a more than twofold increase in urinary tract lesions in laparoscopic hysterectomy as compared with abdominal hysterectomy [1]. To date, it is not known whether this is related to the type of laparoscopic hysterectomy, the size of the uterus, the surgeons' experience, the sealing technique or other influencing factors. Also, there is no evidence that a LSH procedure carries a lower risk of complications as compared with TLH. Morelli conducted a prospective randomised trial comparing complications and clinical outcome following LSH and TLH and found no difference in complication rate [10]. In a prospective observational study, Makinen has shown that with increasing experience in laparoscopic hysterectomy, the urinary tract injury rate decreases to a rate similar to abdominal hysterectomy [11].

Although the relatively large sample size is the strength of this study, we are well aware that the retrospective study design is a major limitation. Nevertheless, this study is the first to compare the short-term and long-term postoperative outcomes of LSH with all subtypes of LH.

In conclusion, the present regional audit of the last 10 years of laparoscopic hysterectomies shows that a LSH as compared with the different types of LH was associated with less short-term complications but more long-term complications.
Open Access This article is distributed under the terms of the Creative Commons Attribution Noncommercial License which permits any noncommercial use, distribution, and reproduction in any medium, provided the original author(s) and source are credited.

\section{References}

1. Nieboer TE, Johnson N, Lethaby A, Tavender E, Curr E, Garry R, van Voorst S, Mol BW, Kluivers KB (2009) Surgical approach to hysterectomy for benign gynaecological disease. Cochrane Database Syst Rev (3):CD003677

2. Garry R, Fountain J, Mason S, Hawe J, Napp V, Abbot J et al (2004) The eVALuate study: two parallel randomized trials, one comparing laparoscopic with abdominal hysterectomy, the other comparing laparoscopic with vaginal hysterectomy. BMJ 328:1229-1236

3. Kluivers K, Hendriks J, Mol BW, Bongers M, Bremer G, de Vet H (2007) Quality of life and surgical outcome after total laparoscopic versus total abdominal hysterectomy for benign disease; a randomize controlled trial. J Minim Invasive Gynecol 14:145-152

4. Lethaby A, Ivanova A, Johnson N (2006) Total versus subtotal hysterectomy for benign gynaecological conditions (review). Cochrane Database Syst Rev (2):CD004993

5. Lieng M, Qvigstad E, Ballard K (2008) Long term outcome following laparoscopic supracervical hysterectomy. BJOG 115:1605-1610

6. Reich H, Roberts T (2003) Laparoscopic hysterectomy in current gynaecological practice. Rev Gynaecol Prac 3:32-40

7. Donnez O, Jadoul P, Squifflet J, Donnez J (2009) A series of 3190 laparoscopic hysterectomies for benign disease from 1990 to 2006: evaluation of complications compared with vaginal and abdominal procedures. BJOG 16(4):492-500

8. Nisolle M, Donnez J (1997) Subtotal hysterectomy in patients with endometriosis, an opinion. Fertil Steril 67:1185-1187

9. El Toukhy TA, Hefni M, Davies A, Mahadevan S (2004) The effect of different kinds of hysterectomy on urinary and sexual function: a prospective study. J Obstet Gynaecol 24:420-425

10. Morelli M, Noia R, Chiodo D, Mocciaro R, Amendola G, Zullo F et al (2007) Laparoscopic supracervicale hysterectomy versus laparoscopic total hysterectomy: a prospective randomized study. Minerva Gynecol 59(1):1-10

11. Makinen J, Johansson J, Tomas C, Tomas E, Heinonen PK, Laatkainen $\mathrm{T}$ et al (2001) Morbidity of 10110 hysterectomies by type of approach. Hum Reprod 16(7):1473-1478 